COLO-HEP-375

hep-th/9609211

September 1996

\title{
Anomaly cancellation in M-theory
}

\author{
S. P. de Alwis内 \\ Department of Physics, Box 390, University of Colorado, Boulder, CO 80309
}

\begin{abstract}
We show the complete cancellation of gauge and gravitational anomalies in the Mtheory of Horava and Witten using their boundary contribution, and a term coming from the existence of two and five-branes. A factor of three discrepancy noted in an earlier work is resolved. We end with a comment on flux quantization.
\end{abstract}

\footnotetext{
1e-mail: dealwis@gopika.colorado.edu
} 
In the M-theory of Horava and Witten [1] several consistency checks associated with anomaly cancellation were verified. However one test which involved a numerical coefficient of a purely gravitational anomaly was not carried out. In an earlier paper by the author [2] the coefficient of a certain M-theory Green-Schwarz term [3] was determined, but there appeared to be a factor of three discrepancy with the expression (3.12) of [1]. In this short note we review the anomaly cancellation argument and find that the M-theory topological terms do indeed cancel both gauge and gravitational anomalies. Finally we comment on flux quantization in M-theory in the light of a recent paper by E. Witten [4].

We work with the "downstairs" version of the theory, i.e. on an 11-D manifold $M=M_{10} \times S^{1} / Z_{2}$. The topological term in the low energy effective action of M-theory is

$$
-\frac{1}{\kappa^{2}} \frac{1}{6} \int_{M} C \wedge K \wedge K
$$

In the abovet $C$ is the three form gauge field of $11 \mathrm{D}$ supergravity and $K=d C$. In the Horava-Witten theory the the manifold $M$ has a boundary which consists of two disconnected components on each of which $E_{8}$ gauge fields live, so that on dimensional reduction to ten dimensions one gets the low energy effective action of the Heterotic $E_{8} \times E_{8}$ theory. On checking the supersymmetry of the resulting theory it was found by

\footnotetext{
${ }^{1}$ In this paper it was also found that the dimensionless ratio of gauge and gravitational couplings determined by anomaly cancellation in [1] (see below) checks with a result obtained from string dualtiy and D-brane methods, thus giving an additional test of M-theory.

${ }^{2}$ It should be noted that (0.1) has a factor two compared to the usual term since we are working in the "downstairs" version of the theory with $M=M_{10} \times S^{1} / Z_{2}$ where the integral goes over half the volume of the "upstairs" version where $M=M_{10} \times S^{1}$ and the fields are $Z_{2}$ symmetric.

${ }^{3}$ Our 11 D supergravity conventions and definitions are the same as in [3]. In particular we define a p-form gauge field as $A=\frac{1}{p !} A_{I_{1} \ldots I_{p}} d x^{I_{1}} \ldots d x^{I_{p}}$. The field strength is then $F=d A$ or in components $F_{I_{1} \ldots I_{p+1}}=(p+1) \partial_{\left[I_{1}\right.} A_{\left.I_{2} \ldots I_{p+1}\right]}$ with unit strength anti-symmetrization. The comparison with the notation of Horava and Witten is as follows. $K=\sqrt{2} G, C=\sqrt{2} C^{H W}$ where $C^{H W}=C_{I J K}^{H W} d x^{I} d x^{J} d x^{K}, G^{H W}=d C^{H W}=\frac{1}{4 !} G_{I J K L} d x^{I} d x^{J} d x^{K} d x^{L}$ are the three form gauge field and field strength (called $\mathrm{C}$ and $\mathrm{G}$ in [1]) as defined by Horava and Witten. Our indices I,J,K,L, run from 1 to 11 whilst indices A,B,C,D run from 1 to 10 .
} 


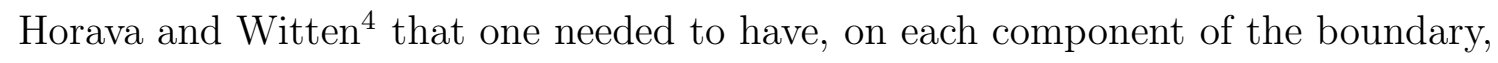

$$
\left.K\right|_{\partial M}=\frac{\kappa^{2}}{2 \lambda^{2}} \hat{I}_{4}
$$

In the above $\lambda$ is the gauge field coupling,

$$
\hat{I}_{4}=\frac{1}{2} \operatorname{tr} \mathcal{R}^{2}-\operatorname{tr} F^{2},
$$

$F$ is an $E_{8}$ gauge field strength, and $\mathcal{R}$ is the curvature two form. Now defining $Q_{3}=$ $\frac{1}{2} \omega_{3 L}-\omega_{3}$, where the two omegas correspond to the Lorentz and gauge Chern-Simons forms, we have the standard descent equations, ,

$$
\hat{I}_{4}=d Q_{3}, \quad \delta Q_{3}=d Q_{2}^{1} .
$$

where $\delta$ is a gauge and local Lorentz variation and $Q_{2}^{1}$ is a two-form that is linear in the gauge parameter.

From (0.2), the relation $K=d C$, and the first equation in (0.4), we have (up to an irrelevant exact form)

$$
\left.C\right|_{\partial M}=\frac{\kappa^{2}}{2 \lambda^{2}} Q_{3} .
$$

Hence from the second equation in (D.4)

$$
\left.\delta C\right|_{\partial M}=\frac{\kappa^{2}}{2 \lambda^{2}} d Q_{2}^{1}
$$

Now clearly we may extend this variation to the bulk by writing

$$
\delta C=d \Lambda,\left.\quad \Lambda\right|_{\partial M}=\frac{\kappa^{2}}{2 \lambda^{2}} Q_{2}^{1} .
$$

Hence we have from (0.1) , and (0.7)

$$
\begin{aligned}
\delta W & =-\frac{1}{\kappa^{2}} \frac{1}{6} \int_{M} d \Lambda \wedge K \wedge K \\
& =-\frac{1}{\kappa^{2}} \frac{1}{6}\left(\frac{\kappa^{2}}{\lambda^{2}}\right)^{3} \frac{1}{2} \int_{\partial M} Q_{2}^{1} \wedge \frac{\hat{I}_{4}^{2}}{4},
\end{aligned}
$$

\footnotetext{
${ }^{4}$ See equation (2.20) of the second paper of [1].
}

${ }^{5}$ See for example reference [6], chapter 13. 
where to get the second equality we have used Stokes' theorem, $d K=0$, and (0.2).

Now the boundary theory is anomalous and the variation of the quantum effective action $\Gamma$ is given by

$$
\delta \Gamma=-\frac{1}{48(2 \pi)^{5}} \int_{\partial M} Q_{2}^{1} \wedge\left(-\frac{\hat{I}_{4}^{2}}{4}+X_{8}\right),
$$

where $X_{8}=-\frac{1}{8} \operatorname{tr} \mathcal{R}^{4}+\frac{1}{32}\left(\operatorname{tr} \mathcal{R}^{2}\right)^{2}$. Cancellation of the $\hat{I}_{4}^{2}$ part of the anomaly then determines

$$
\eta^{-1} \equiv \frac{\kappa^{4}}{\lambda^{6}}=\frac{1}{4(2 \pi)^{5}}
$$

as in [1].

Now as shown in [3] the existence of two and five branes in the theory implies that there is an additional topological (Green-Schwarz) term in M-theory. This is given by,

$$
W_{5}=\left(\frac{(2 \pi)^{2}}{2 \kappa^{2}}\right)^{1 / 3} \frac{1}{24(2 \pi)^{4}} 2 \int_{M} C \wedge X_{8} .
$$

The first factor in the equation above was obtained from the relation $T_{2}=\left[\frac{(2 \pi)^{2}}{2 \kappa^{2}}\right]^{1 / 3}$, which was originally determined using D-brane methods [2], but after correcting a factor of two in the quantization formula of [3] as discussed in the appendix to [2], it can also be fixed purely within M-theory.?

Using equations (0.7), Stokes' theorem and $d X_{8}=0$ we have

$$
\begin{aligned}
\delta W_{5} & =\left(\frac{(2 \pi)^{2}}{2 \kappa^{2}}\right)^{1 / 3} \frac{1}{24(2 \pi)^{4}} 2 \int_{M} d \Lambda \wedge X_{8} \\
& =\frac{1}{48(2 \pi)^{5}} \int_{\partial M} Q_{2}^{1} \wedge X_{8} .
\end{aligned}
$$

${ }^{6}$ The numerical coefficient in $(0.9$ ) is fixed by standard methods. See for example [6] equation (13.3.41), (13.4.5) the line before (13.5.6) and equations (13.5.5) and (13.5.8). The form of the anomaly is given in [1].

${ }^{7}$ The existence of this term may also be inferred from an earlier string theory calculation [5].

${ }^{8}$ There is an extra factor of 2 compared to equation (0.20) of [2] because we are in the "downstairs" theory - see footnote 2 . 
In the last equation we have used the value of $\eta$ given in (0.10). Thus we have the complete cancellation of the anomalies in the Horava-Witten M-theory,

$$
\delta W+\delta W_{5}+\delta \Gamma=0 .
$$

While the above was being written up, a paper by E. Witten appeared [4 in which, inter alia, some issues of normalization in M-theory were discussed using index theory. To conclude this note we would like to make some related comments. Equation (0.2) may be rewritten (after using 0.10) as

$$
\left.\left.\frac{G}{2 \pi}\right|_{\partial M} \equiv\left[\frac{(2 \pi)^{2}}{2 \kappa^{2}}\right]^{1 / 3} \frac{K}{2 \pi}\right|_{\partial M}=w(V)-\frac{\lambda}{2},
$$

where $w=F \wedge F /\left(16 \pi^{2}\right)$ has integer valued periods, being the second Chern class of the $E_{8}$ bundle, and $\lambda \equiv p_{1} / 2=\mathcal{R} \wedge \mathcal{R} /\left(16 \pi^{2}\right)$ which is half the Pontryagin class $p_{1}$ of the tangent bundle, also has integer-valued periods for a spin manifold so that in general as pointed out in [4], $G /(2 \pi)$ has half integer periods . By considering a 4-cycle $(C)$ in the bulk that is homologous to one in the boundary $\left(C^{\prime}\right)$ this result was extended in [4] to the statement

$$
\int_{C}\left(\frac{G}{2 \pi}-\frac{\lambda}{2}\right) \in \mathcal{Z}
$$

There is an alternate way to get the normalization of $G /(2 \pi)$. This follows from the fact that $K=d C$ and $C$ is the three form field coupling to the 2-brane of M-theory. In earlier work this was given as $T_{2} \int_{C} K / 2 \pi \in \mathcal{Z}$ but due to anomalies of fermionic determinants in odd dimensions, it was pointed out in 4 that this gets modified to

$$
\int_{C}\left(\frac{T_{2} K}{2 \pi}-\frac{\lambda}{2}\right) \epsilon \mathcal{Z}
$$

The consistency of the two normalizations is then a consequence of the relation $T_{2}=$ $\left[\frac{(2 \pi)^{2}}{2 \kappa^{2}}\right]^{1 / 3}$ derived in [2]. Note that this is a check on the consistency of M-theory that is ${ }^{9}$ see for example [3] and the appendix of [2]. 
independent of the check coming from the pure gravity anomaly cancellation. TH

\section{Acknowledgments}

I wish to thank E. Witten for the suggestion to check M-theory consistency conditions, and S. Chaudhuri and P. Horava for discussions. This work is partially supported by the Department of Energy contract No. DE-FG02-91-ER-40672.

\section{References}

[1] P. Horava and E. Witten, Nucl. Phys. B460 (1996) 506, hep-th/9510209, and hepth/9603142.

[2] S. P. de Alwis, hep-th/9607011 (Phys. Lett. B - in press).

[3] M.J. Duff, J.T. Liu, and R. Minasihan, Nucl. Phys. BB452 261 (1995) hepth/9506126.

[4] E. Witten, hep-th/9609122.

[5] C. Vafa and E. Witten, Nucl. Phys. B463 (1995) 261, hep-th/9505053.

[6] M.B. Green, J. Schwarz, and E. Witten, "Superstring theory" (Cambridge 1987).

\footnotetext{
${ }^{10}$ As noted in [2] (equation (8), (11) and the discussion after the latter equation), the calculation of $T_{2}$ can be done in a fashion that is completely independent of M-theory quantization conditions.
} 